\title{
Human visual gamma for color stimuli: When LGN drive is equalized, red is not special
}

\author{
Benjamin Johannes Stauch ${ }^{1,2,3}$, Alina Peter ${ }^{1,2}$, Isabelle Ehrlich ${ }^{1,4}$, Zora Nolte ${ }^{1}$, Pascal Fries ${ }^{1,5 *}$ \\ ${ }^{1}$ Ernst Strüngmann Institute (ESI) for Neuroscience in Cooperation with Max Planck Society, \\ 60528 Frankfurt, Germany \\ ${ }^{2}$ International Max Planck Research School for Neural Circuits, 60438 Frankfurt, Germany \\ ${ }^{3}$ Brain Imaging Center, Goethe University Frankfurt, 60528 Frankfurt, Germany \\ ${ }^{4}$ Department of Psychology, Goethe University Frankfurt, 60323 Frankfurt, Germany \\ ${ }^{5}$ Donders Institute for Brain, Cognition and Behaviour, Radboud University Nijmegen, 6525 EN \\ Nijmegen, Netherlands
}

*Correspondence: pascal.fries@esi-frankfurt.de

\section{SUMMARY}

Strong gamma-band oscillations in primate early visual cortex can be induced by spatially homogeneous, high-contrast stimuli, such as color surfaces. Compared to other hues, particularly strong gamma oscillations have been reported for red stimuli. However, precortical color processing and the resultant strength of input to $\mathrm{V} 1$ has often not been fully controlled for. This leaves the possibility that stronger responses to some hues were due to differences in V1 input strength. We presented stimuli that had equal luminance and color contrast levels in a color coordinate system based on color responses of the lateral geniculate nucleus, the main input source for area V1. With these stimuli, we recorded magnetoencephalography in 30 human subjects. We found narrowband color-induced gamma oscillations in early visual cortex, which, contrary to previous reports, did not differ between red and green stimuli of equal L-M cone contrast. Notably, blue stimuli with contrast exclusively on the S-cone axis induced very weak gamma responses, as well as smaller event-related fields and poorer change detection performance. The strength of human color gamma responses could be well explained by the strength of thalamic input induced by each hue and does not show a clear red bias when this input strength is properly equalized. 


\section{INTRODUCTION}

In early visual cortex of human and non-human primates, visual stimuli often induce rhythmic activity in the gamma band (Hoogenboom et al., 2006; Jia et al., 2011; Kreiter and Singer, 1992). Gamma oscillations and their coherence between neuronal populations have been related to attentional selection (Bauer et al., 2006; Bosman et al., 2012; Fries et al., 2001; Magazzini and Singh, 2018), directed information flow (Bastos et al., 2015; Besserve et al., 2015; Michalareas et al., 2016; van Kerkoerle et al., 2014), stimulus learning (Brunet et al., 2014; Peter et al., 2020; Stauch et al., 2021), and further cognitive functions (for reviews, see Fries, 2015; Singer, 2018).

In recent years, research on visually induced gamma has increasingly focused on the fundamental question of which visual stimulus features determine gamma amplitude. Especially strong gamma oscillations have been observed for high-contrast black and white stimuli that extend beyond the size of single-neuron receptive fields (Gieselmann and Thiele, 2008; Jia et al., 2011) and whose features are contiguous over space (Brunet and Fries, 2019; Hermes et al., 2019; Lowet et al., 2017; Uran et al., 2020; Vinck and Bosman, 2016). Likely, this dependence exists because extensive and uniform stimuli synchronize larger populations of neurons into a common gamma rhythm, thereby generating a clearer oscillatory population signal (Gray et al., 1989; Lowet et al., 2015; Lowet et al., 2017).

Large homogenous patches of color have also been shown to induce strong gamma oscillations, both in the early visual cortex of macaques (Brunet et al., 2015; Peter et al., 2019; Rols et al., 2001; Shirhatti and Ray, 2018) and humans (Bartoli et al., 2019; Li et al., 2021; Perry et al., 2020). Interestingly, red stimuli have been reported to induce especially strong gamma-band responses in several of these reports (Bartoli et al., 2019; Perry et al., 2020; Rols et al., 2001; Shirhatti and Ray, 2018). It has been speculated that this might be due to a higher ecological relevance of the color red in natural environments (Bartoli et al., 2020), and it was recently related to potentially faster adaptation of the M-cone pathway (Peter et al., 2019). However, when comparing across different stimuli, the strength of input to the neuronal populations generating the investigated gamma signal should be controlled, because gamma power scales with the strength of excitatory synaptic input (Hadjipapas et al., 2015; Henrie and Shapley, 2005; Lewis et al., 2021). Color-induced gamma has mostly been studied in area V1 (see above). In primates, feedforward driving input to V1 originates mostly from the LGN (Felleman and Van Essen, 1991; Sherman, 2005), including for color stimuli (Chatterjee and Callaway, 2003; Shapley, 2019). Note that in awake primates, the LGN itself does not show visually induced gamma (Bastos et al., 2014; tested with grating stimuli), such that V1 is likely the first stage in the primate visual system generating gamma.

To show color stimuli in coordinates that model LGN activation (and thereby V1 input), monitor light emission spectra can be measured to define the strength of S-, M-, and L-cone excitation they induce. Then, stimuli and stimulus background can be picked from an equiluminance plane in DKL space (Derrington, Krauskopf, and Lennie, 1984), whose axes represent L-M and S-(L+M) cone contrast values encoded in the LGN. Because spectral peaks of cone opsin sensitivity show variability over individuals (Neitz and Jacobs, 1986; Neitz and Neitz, 2011), recruiting a larger sample than is possible in human patient or macaque studies is necessary to measure the general, population-wide relationship between stimulus color and induced gamma oscillations in the early visual cortex.

In this study, we recorded MEG in 30 human participants while we presented them with uniform, circular color disks picked from a DKL equiluminance plane on an equiluminant background. Crucially, 
their cone contrasts on the L-M and the S- $(\mathrm{L}+\mathrm{M})$ axes were equalized. We analyzed the MEG recordings at the source level (Gross et al., 2001; Van Veen et al., 1997) and found that color-induced gammaband responses localized to early visual cortex with a peak in area V1, confirming that color-gamma responses can be studied non-invasively in humans. With colors that had identical luminance and identical color contrast in DKL space, we could not confirm stronger gamma responses for red stimuli. Instead, equiluminant colors with comparable absolute cone contrasts on the L-M axis induced gamma-power responses of equal strength in area V1, independent of their redness. Colors on the S$(L+M)$ axis induced weaker gamma power, especially for colors strongly activating only the S-cone.

\section{RESULTS}

Trial structure, stimuli and stimulus color coordinates are shown in Figure 1 and described in detail in the Methods. In brief, participants fixated a central fixation spot on a grey background. After a $1.2 \mathrm{~s}$ baseline, they were presented with a stimulus disk that was centered on the fixation spot and had a diameter of 10 deg visual angle (dva). The disk was filled either with uniform color (Figure 1A) or with a luminance grating (Figure 1D). After 0.3-2 s, a change occurred at a random position on the disk, with the shape of a Gaussian blob ( 3.7 dva diameter). For colored disks, the change was a small decrement in color contrast, for gratings a small decrement in luminance contrast, in both cases staircased per participant and color/grating to $85 \%$ correct detection performance. Subjects then speedily reported the side of the contrast decrement relative to the fixation spot using a button press. Feedback was given as a smiley on correct trials, then the next trial commenced upon fixation.

The eight colors were sampled from an ellipse on a DKL equiluminance plane, such that they were DKL-equiluminant to each other and to the background (Figure 1C). Color coordinates are given in Figure 1-figure supplement 1. To simplify reporting, we will refer to colors on the L-M axis as "red" and "green", to colors on the S-(L+M) axis as "blue" and "yellow", and to colors with components from both axes as "red-blue", "red-yellow", "green-blue" and "green-yellow". Note that these labels do not fully correspond to subjective perceptual labels: For example, monochromatic light strongly activating S-cones but not L-/M-cones would look purple to the observer, as can be seen from the spectral sensitivities of the S/M/L-cones (Fig. 1B, Stockman and Sharpe, 2000).

Note that the colors were constrained to equalized cone contrast levels, while the grating was chosen to generate a maximally strong gamma signal for comparison. Differences in the strength of neuronal responses between grating and colors are therefore difficult to interpret, because it is unclear how input strength could be equalized. 


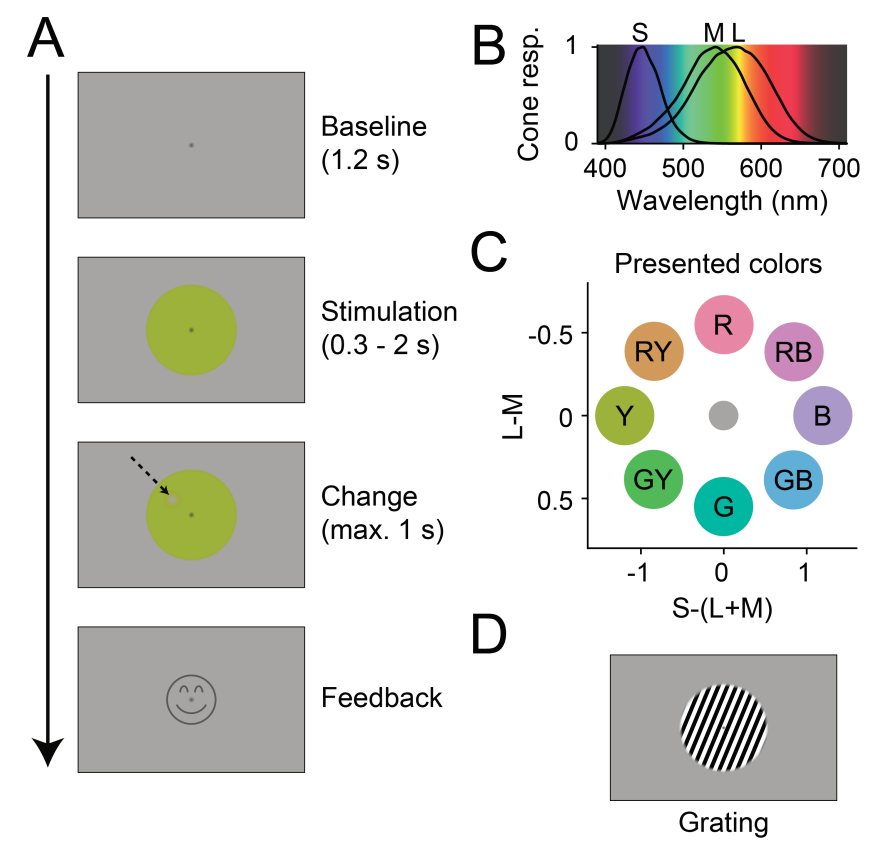

Figure 1. Experiment design: (A) Temporal structure of one trial. After fixation onset, a grey baseline was shown for $1.2 \mathrm{~s}$, followed by $0.3-2 \mathrm{~s}$ of stimulation with a stimulus disk. Then, a change spot appeared at a random position on the disk (indicated here with an arrow, not visible in the actual experiment), which the participants needed to report. Upon correct report, a smiley was shown. (B) Human cone sensitivity spectra (Stockman and Sharpe, 2000) plotted on an estimate of perceptual wavelength color. (C) DKL coordinates for all eight stimulus colors shown in this study, relative to the background. Letters indicate stimulus labels used in Results. Note that color hues of these color disks, when displayed on a non-calibrated monitor or printout, will not fully correspond to the hues shown in the study. (D) To compare the strength of induced gamma responses between color and grating stimuli, some trials showed a grating instead of a color stimulus.

\section{Subjects show hue-dependence in reaction times and detection thresholds}

As set by the staircase, participants' report accuracy was close to $85 \%$ (Grating: $83 \%, \mathrm{Cl}_{95 \%}=[76 \% 87 \%]$, Colors: $\left.87 \%, \mathrm{Cl}_{95 \%}=[86 \% 87 \%]\right)$. However, change detection performance, as defined by the color contrast of the stimulus change on which the per-color staircases converged, differed across hues $\left(F(7,203)=82.18, p<3 * 10^{-16}\right)$. Participants reached $85 \%$ accuracy with a change color contrast towards background between $20 \%\left(\mathrm{Cl}_{95 \%}=[17 \%, 23 \%]\right)$ for red-yellow and $37 \%\left(\mathrm{Cl}_{95 \%}=[31 \%, 45 \%]\right)$ for red-blue stimuli (Figure 2D). There was one notable outlier: For blue stimuli, the necessary change color contrast towards background was $71 \%\left(\mathrm{Cl}_{95 \%}=[66 \%, 76 \%]\right)$, significantly higher than for other hues $\left(t(29)=18.01, p<3^{*} 10^{-16}\right)$.

On average, participants took $496 \mathrm{~ms}$ ( $\mathrm{Cl}_{95 \%}=$ [467 ms, $524 \mathrm{~ms}$ ], all confidence intervals based on nonparametric bootstraps) to report the change location on grating stimuli. For color stimuli, they took on average $547 \mathrm{~ms}\left(\mathrm{Cl}_{95 \%}=[521 \mathrm{~ms}, 570 \mathrm{~ms}]\right)$. Across the different color hues, reaction times differed $\left(F(7,203)=9.26, p<7^{*} 10^{-10}\right)$. Subjects were quickest to detect contrast changes on red stimuli (Mean $=509 \mathrm{~ms}, \mathrm{Cl}_{95 \%}=[483 \mathrm{~ms}, 540 \mathrm{~ms}]$ ), and slowest for green-yellow stimuli (Mean = $562 \mathrm{~ms}$, $\mathrm{Cl}_{95 \%}=[535 \mathrm{~ms}, 587 \mathrm{~ms}]$, Figure 2-figure supplement 1).

\section{ERFs are weakest for blue stimuli}

Both grating stimuli and equiluminant color disks induced clear visual event-related fields (ERFs) in area V1 (Figure 2A), with a shape and timing similar to visually evoked potentials recorded in macaque V1 (Rols et al., 2001; Fig. 5B). ERFs were z-scored relative to the per-trial baseline (see Methods). The ERF differed across stimuli during a prominent component, 57-94 ms post stimulus onset 

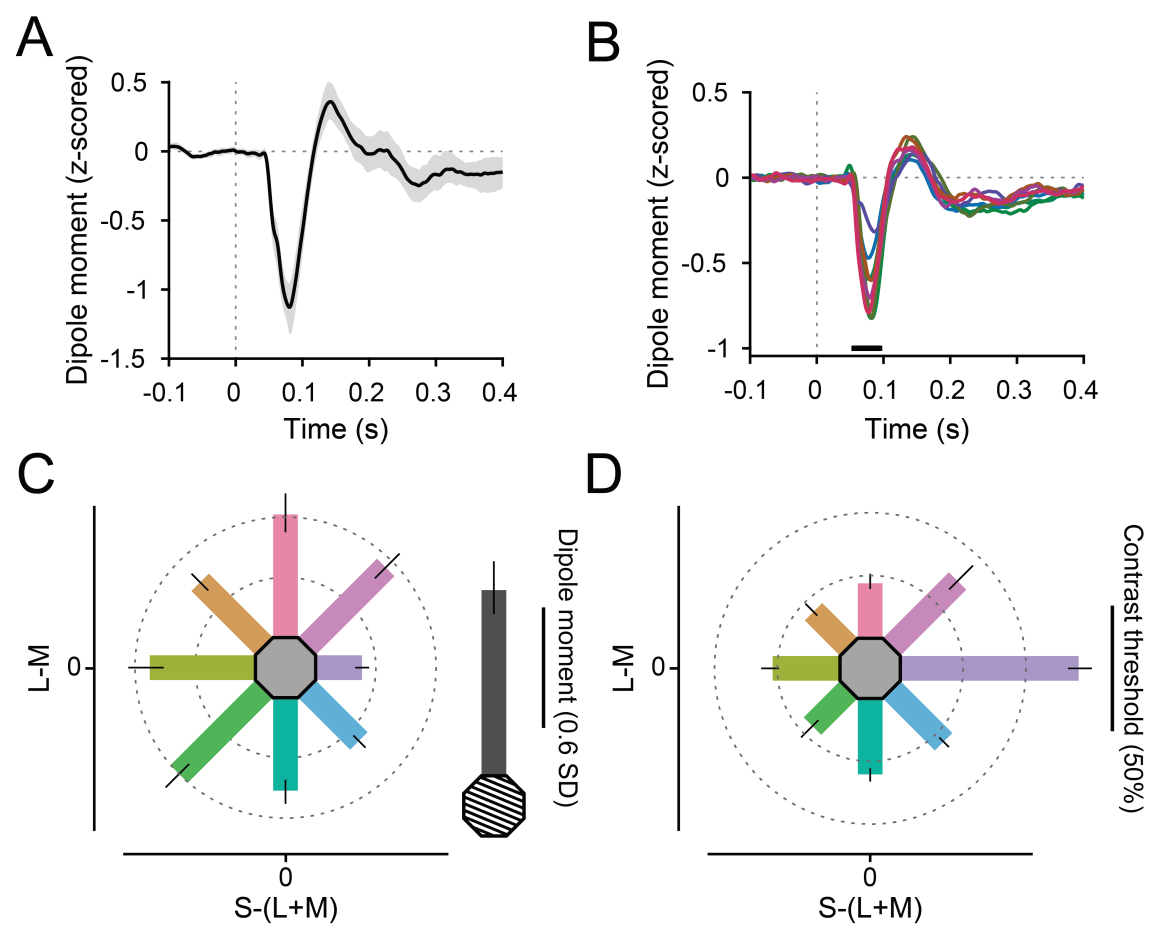

Figure 2. Event-related field responses: (A) Grating-evoked ERF, averaged over V1 dipoles and participants. Error region shows $95 \% \mathrm{Cl}$ over participants. (B) Color-evoked ERFs, averaged over V1 dipoles and participants. Black bar indicates significant differences across colors, $t_{\max }$-corrected for multiple comparisons. Line colors reflect stimulus colors, but have been darkened for readability. (C) ERF dipole moment 70-90 ms post-stimulus onset (relative to baseline variability), averaged over V1 dipoles and participants. Error bars represent $95 \% \mathrm{Cls}$ over participants, bar orientation represents stimulus orientation in DKL space. In grey, the average grating-induced ERF dipole moment is shown for comparison. (D) Average relative contrast steps towards the background color needed to achieve $85 \%$ target detection accuracy for all stimuli. Error bars represent $95 \% \mathrm{Cls}$ over participants, bar orientation represents stimulus orientation in DKL space. For C-D, the length of the scale bar corresponds to the distance from the edge of the hexagon to the outer ring.

(Figure $\left.2 \mathrm{~B}, \mathrm{~F}(3.43,98.95)=32.94, \mathrm{p}_{\mathrm{GG}}<3 * 10^{-12}\right)$, which we suspect to be of similar origin as the N70 component recorded in Rols et al. (2001). The N70 component was stronger for grating compared to color stimuli (Figure $2 \mathrm{C}, \mathrm{t}(29)=6.94, p<2 * 10^{-7}$ ). Across stimulus hues, the N70 component was strongest for red (Mean $=-0.60, \mathrm{Cl}_{95 \%}=[-0.50,-0.69]$ ) and green-yellow stimuli (Mean $=-0.59$, $\left.\mathrm{Cl}_{95 \%}=[-0.51,-0.67]\right)$, and weakest for blue stimuli (Mean $\left.=-0.23, \mathrm{Cl}_{95 \%}=[-0.20,-0.26]\right)$.

\section{Color stimuli induce V1 gamma oscillations measurable in MEG}

In addition, color and grating stimuli induced significant visual narrowband gamma-band power increases in early visual cortex (Figure 3A-D, F). Color stimuli induced gamma-power increases at the subject-specific gamma peak of on average $19 \%\left(\mathrm{Cl}_{95 \%}=[14 \% 26 \%]\right)$ relative to the baseline, whereas grating stimuli induced gamma power increases of $100 \%\left(\mathrm{Cl}_{95 \%}=[80 \% 120 \%]\right.$, $\mathrm{t}_{\text {difference }}(29)=8.32$, $\left.\mathrm{p}<4 * 10^{-9}\right)$. 
bioRxiv preprint doi: https://doi.org/10.1101/2021.11.22.469550; this version posted November 22, 2021. The copyright holder for this preprint (which was not certified by peer review) is the author/funder, who has granted bioRxiv a license to display the preprint in perpetuity. It is made available under aCC-BY 4.0 International license.

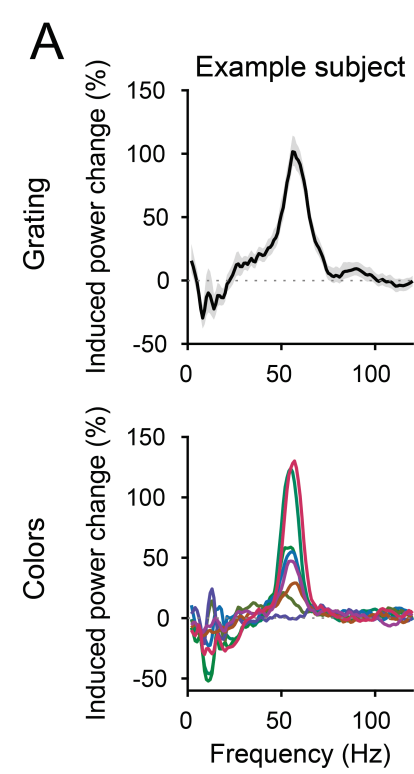

$E$

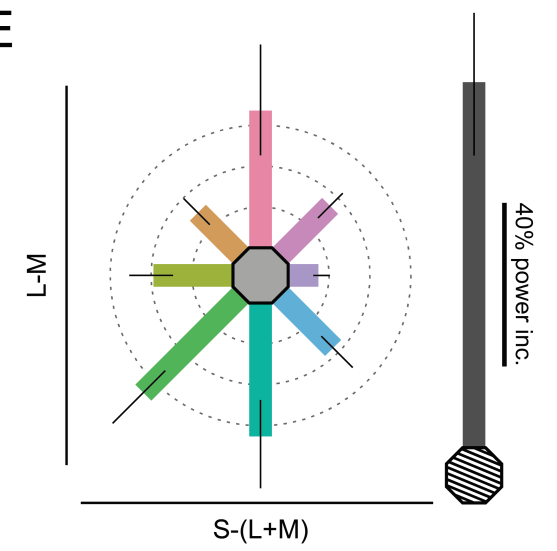

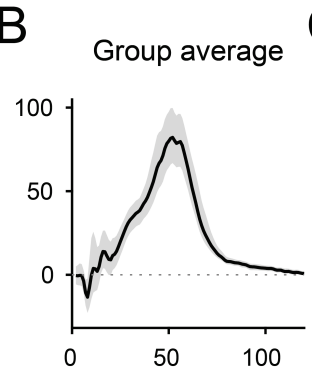

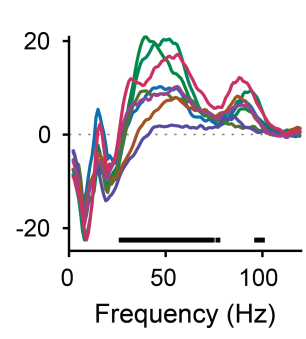

$\mathrm{F}$
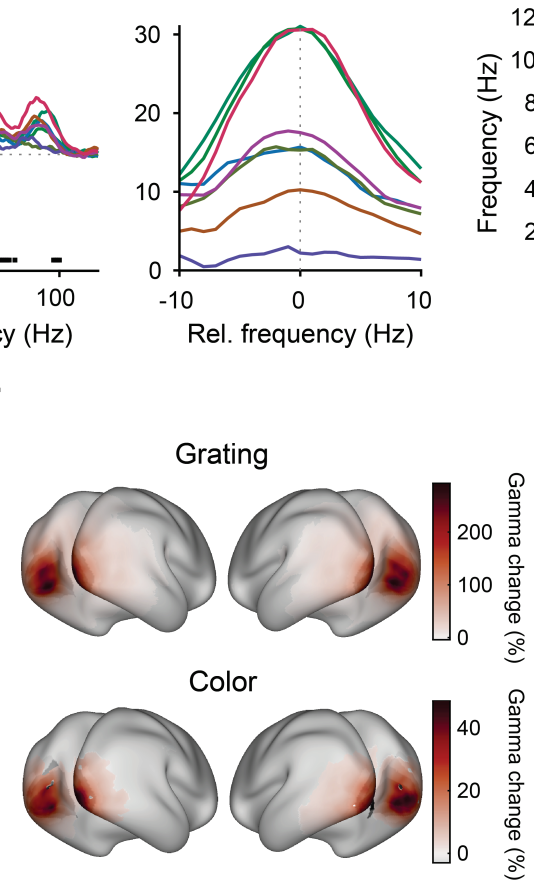
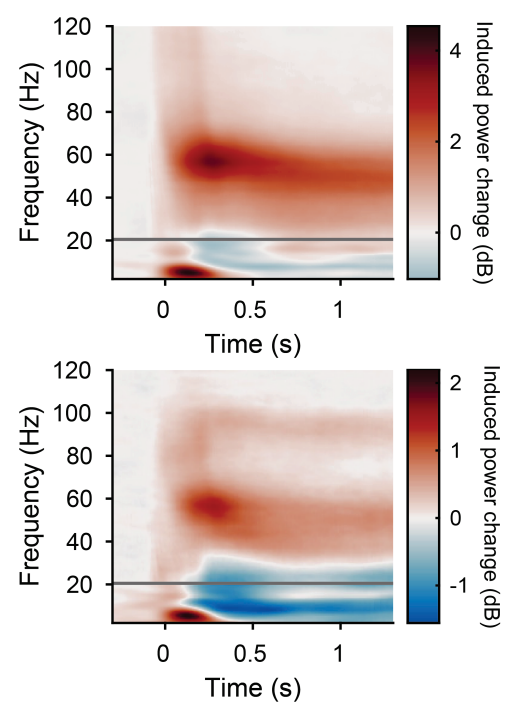

G

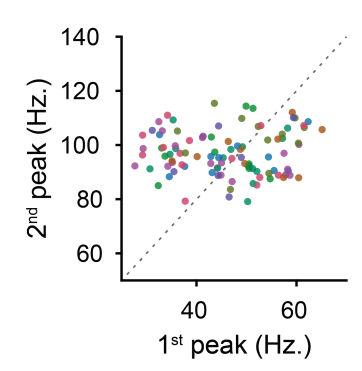

Figure 3. Gamma-band responses: (A) Stimulus-induced power changes over baseline for one example participant that showed strong gamma-band responses (averaged over V1 dipoles). Top: Grating-induced power changes. Error region shows 95\% Cl over trials. Bottom: Color-induced power changes. (B) Same as A, but averaged over participants. Error region shows $95 \% \mathrm{Cl}$ over participants. (C) Same as B, but peak-aligned before averaging. For A-C, line colors reflect stimulus colors, but have been darkened for readability. (D) Average stimulus-induced power change in V1 as a function of time and frequency. Top: For grating stimuli. Bottom: For green stimuli. (E) Average stimulus-induced gamma power change for all stimuli. Error bars represent $95 \% \mathrm{Cls}$ over participants, bar orientation represents stimulus orientation in DKL space. In grey, the average grating-induced gamma power strength is shown for comparison. The length of the scale bar corresponds to the distance from the edge of the hexagon to the outer ring. (F) Average stimulus-induced gamma-power change (individual gamma peak $\pm 10 \mathrm{~Hz}$ ), source-projected to all cortical dipoles. Values are significance-masked using false discovery rate control (Benjamini and Yekutieli, 2001), black-to-white shading indicates areas V1, V2, V3, V3A, and V4. (G) Gamma peak frequencies of the first and second gamma peak for all participant-color combinations in which a first and a second gamma peak was found. Dot color corresponds to stimulus color. Dotted line indicates the expected frequency relationship, if $1^{\text {st }}$ and $2^{\text {nd }}$ peak frequencies were harmonics of each other.

Grating- and color-induced gamma source-localized to similar sources, being strongest in areas V1/V2 and extending into parietal and temporal cortex (Figure 3F). For both grating and color stimuli, induced gamma-power changes were strongest in areas V1 (grating: 142\%; color: 21\%), V2 (grating: 131\%, color: 19\%), V3 (grating: 113\%, color: 19\%), and V4 (grating: $91 \%$, color: $17 \%)$ respectively.

The induced change spectra were fit per participant and stimulus with the sum of a linear slope and up to two Gaussians. $97 \%$ of all participants showed a significant gamma peak for at least one color and for the grating stimulus. A second, higher gamma peak was detected in $90 \%$ of participants for at 
least one color stimulus. While induced gamma was five times stronger for grating compared to color stimuli, only $13 \%$ of participants showed a second gamma peak when the grating stimulus was shown. For the two peaks often observed with color stimuli, there was no linear relation between the two peak frequencies over colors and participants $(r=0.06, p=0.58$, Figure $3 \mathrm{G})$, indicating that the second peak was unlikely to be a harmonic of the first. As expected due to interindividual variability in skull thickness, cortical orientation, genetic factors, and peak cone sensitivities (Butler et al., 2019; Neitz and Neitz, 2011; van Pelt et al., 2012; van Pelt et al., 2018), there was substantial variability in colorinduced gamma peak power and frequency over participants (Figure 3-figure supplement 2).

There was a significant difference between the average lower gamma peak frequencies induced by grating $\left(51.6 \mathrm{~Hz}, \mathrm{Cl}_{95 \%}=[49.4 \mathrm{~Hz}, 53.7 \mathrm{~Hz}]\right)$ and color stimuli $(45.9 \mathrm{~Hz}, \mathrm{Cl} 95 \%=[42.6 \mathrm{~Hz}, 49.1 \mathrm{~Hz}]$, $\left.p_{\text {difference }}<3^{*} 10^{-3}\right)$. Across participants, the gamma peak frequencies induced by grating and by color stimuli were correlated $\left(r=0.64, p<3^{*} 10^{-4}\right)$. However, none of the different color pairs showed significantly differing peak frequencies in pairwise comparisons within participants across different colors (Figure 3 -figure supplement $1 \mathrm{~A}$, all $\mathrm{p}_{\mathrm{Holm}}>0.36$ ). The higher peak did not differ in peak frequencies induced by grating $\left(96.5 \mathrm{~Hz}, \mathrm{Cl}_{95 \%}=[91.8 \mathrm{~Hz}, 101.3 \mathrm{~Hz}]\right)$ and color stimuli $(97.1 \mathrm{~Hz}$, $\left.\mathrm{Cl}_{95 \%}=[95.0 \mathrm{~Hz}, 99.1 \mathrm{~Hz}], \quad p_{\text {Difference }}=0.68\right)$ and showed no significant differences in pairwise comparisons across different colors (Figure 3-figure supplement $1 \mathrm{~B}-\mathrm{C}$ all $\mathrm{p}_{\text {Holm }}>0.37$ ).

\section{Induced gamma power does not differ between equiluminant red and green stimuli}

Crucially, for the red and green stimuli that were chosen to have equal absolute L-M contrast, the induced gamma power change was not statistically different between the two (Figure 3E, Red: $30.8 \%$, $\mathrm{Cl}_{95 \%}=[20.3 \%, 43.5 \%]$, Green: $\left.31.2 \%, \mathrm{Cl}_{95 \%}=[21.2 \%, 42.2 \%], \mathrm{t}(29)=0.135, \mathrm{p}_{\text {Holm }}=0.89, \mathrm{BF}_{01}=7.0\right)$. The Bayes factor can be interpreted as meaning that the data are 7.0 times more likely to occur under a hypothesis of no differences between red and green stimuli compared to a hypothesis assuming differences.

On the $S-(L+M)$ axis, stimuli with equal absolute cone contrasts showed differing induced gamma power: While yellow stimuli induced a $15.4 \%$ gamma-power increase over baseline $\left(\mathrm{Cl}_{95 \%}=[10.7 \%, 21.9 \%]\right)$, blue stimuli showed significantly smaller, very weak increases of only $2.2 \%$ $\left(\mathrm{Cl}_{95 \%}=[0.3 \%, 4.3 \%], \mathrm{t}(29)=4.02, \mathrm{p}_{\text {difference }}<0.001\right)$.

\section{Performance, ERF, and induced gamma power are related over colors}

Higher change detection performance (defined as lower final staircased target color contrast) was correlated with stronger average V1 dipole moment (i.e. the negative ERF amplitude during the N70 component) across colors, in the time period from 67 to $93 \mathrm{~ms}$ post stimulus onset (Figure 4A, $r_{\max }=0.43$, all $p_{T_{\max }}<0.03$, corrected for multiple comparisons across time). Higher change detection performance was also correlated with stronger induced $\mathrm{V} 1$ power across colors, for several gamma frequency ranges (Figure $4 \mathrm{~B}$; at $34-37 \mathrm{~Hz}: r_{\max }=-0.27$, all $\mathrm{p}_{\text {Tmax }}<=0.03$; at $39-40 \mathrm{~Hz}: r_{\max }=-0.23$, all $\mathrm{p}_{\text {Tmax }}<0.04$; at $44-53 \mathrm{~Hz}: r_{\max }=-0.31$, all $\mathrm{p}_{\text {Tmax }}<=0.03$, corrected for multiple comparisons across frequency). 

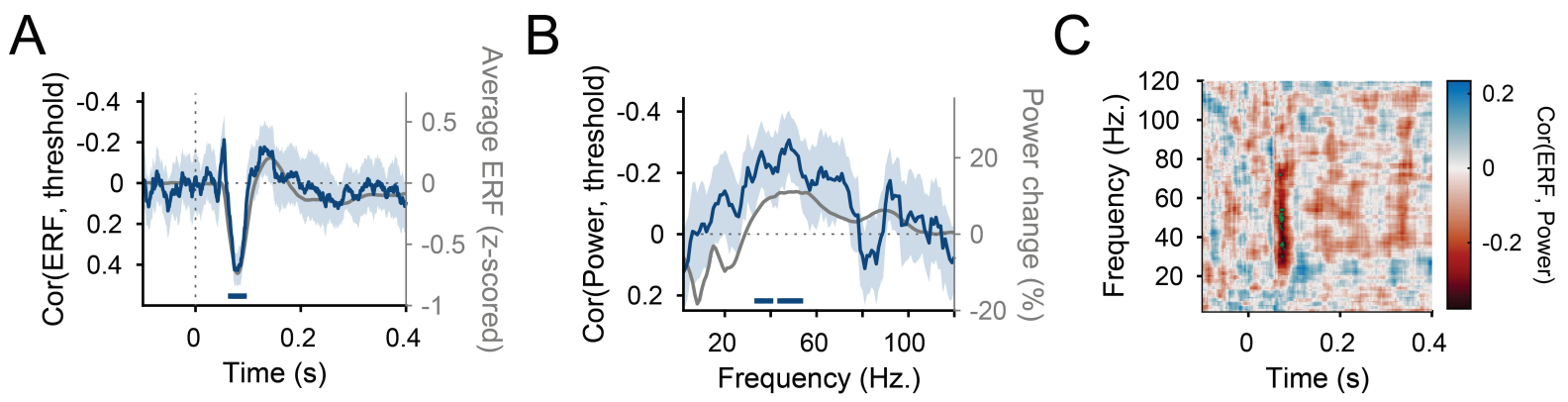

Figure 4. Correlations between ERF, induced power spectra and performance: (A) Correlation, across colors, between V1 ERF dipole moment (averaged over trials and dipoles) and 85\% accuracy threshold. The correlation was first calculated per participant and then averaged over participants. Error-bands represent 95\% Cls over participants. In grey, the average ERF dipole moment timecourse over color stimuli is shown for comparison. Horizontal blue bar represents significant correlation values (multiple comparison-corrected using $t_{\max }$ correction). (B) Same as A, but for the stimulus-induced V1 power change spectra instead of the ERFs. (C) Correlation, across colors, between V1 ERF dipole moment and V1 stimulus-induced power change, as a function of ERF time and spectral frequencies. The correlation was first calculated per participant and then averaged over participants. Significant correlation values (multiple comparison-corrected using $t_{\max }$ correction) are indicated by green dots.

In addition, there were significant correlations between the N70 ERF component and induced gamma power: Across colors, there were several significant correlation clusters between V1 dipole moment 68-79 ms post-stimulus onset and induced power between $28-54 \mathrm{~Hz}$ and $72 \mathrm{~Hz}$ (Figure $4 \mathrm{C}, r_{\max }=-0.30$, $\mathrm{p}_{\text {Tmax }}<0.05$, corrected for multiple comparisons across time and frequency).

\section{DISCUSSION}

In summary, uniform colored stimuli induced gamma oscillations in humans that were measurable in MEG recordings, confirming a previous study (Perry et al., 2020). When red-green color hues were chosen to equalize LGN activation on the L-M axis (and thereby equalize input into V1), the induced V1 gamma power did not differ between red and green hues. Additionally, pure activation of the S- $(L+M)$ axis induced weaker gamma power, especially for color hues activating primarily the S-cone.

This curious asymmetry on the $\mathrm{S}-(\mathrm{L}+\mathrm{M})$ axis could also be seen in smaller amplitudes of early eventrelated field components (N70) in area V1 and in worse color-contrast change-detection performance for high-S (blue) stimuli. Accordingly, across hues, N70 amplitude and gamma power were correlated with color-contrast change-detection performance.

In our data, the color-induced gamma oscillation clearly localized to early visual cortex, was narrowbanded and showed (for $90 \%$ of subjects) a second spectral peak around $80-100 \mathrm{~Hz}$, which did not seem to be a harmonic of the main induced peak and which was rare for grating stimuli.

By designing our stimuli to drive the LGN equally, we could test gamma responses of the early visual cortex for color stimuli of approximately equal V1 input strength on the cardinal DKL axes. While most previous studies of gamma responses to colored stimuli were limited to small samples of non-human primates or human patients, recruiting a bigger sample of human participants allowed us to generalize findings of color gamma responses to the population level (Fries and Maris, 2021).

When comparing our results to previous reports based on intracranial recordings, it is important to note that the MEG signal does not represent a simple average over all population activity underneath the MEG sensor, but is influenced by the location and orientation of the underlying neuronal dipoles and is most sensitive to synchronized neuronal activity (Baillet, 2017; Murakami and Okada, 2006). Thereby, the total power reported here does not correspond to the total power of all oscillatory 
neuronal activity in V1. Additionally, activity sources that are of unequal frequency or phase are likely not fully captured in MEG recordings. On the other hand, intracortical recordings will usually not sample all of area V1 equally, but will emphasize the color tuning of neuronal populations close to the electrodes.

The localization accuracy of source-localized MEG is limited by subject movement, uncertainties in head tracking and source spread. However, with careful head stabilization and exclusion of subjects showing excessive head movements (as practiced here), spatial resolution can be brought down to between 0.45-7 mm, depending on dipole location (Nasiotis et al., 2017). Additionally, the high similarity between early (source-localized) V1 ERF components recorded here and intracortical V1 ERP components recorded in macaque (Rols et al., 2001) suggests that our V1-localized activity in MEG captures the underlying intracortical voltage signal in V1.

\section{Gamma responses to colored stimuli}

In several previous studies in non-human and human primates analyzing early visual cortex gammaband responses to colored stimuli, a red-effect, that is stronger induced gamma power for red vs. nonred colors, was reported. In most of these studies, the presented colors were not equiluminant and did not have equal color contrasts to grey.

In an initial report on recordings in anesthetized macaque V1 and V4, Rols et al. (2001) first described stronger gamma power for a red stimulus compared to a green and a yellow one (heteroluminant, all presented on a gray background). Shirhatti and Ray (2018), recording in awake macaque V1, presented fullscreen, heteroluminant colors after a gray inter-trial background and found strongest gamma power for red and blue hues. When colors were transformed to coordinates based on L- and M-cone activation, induced gamma power was found to increase with cone contrast modulations towards both axis directions in one of the three monkeys tested.

Using natural stimuli, Brunet et al. (2015) presented 65 grayscale and color images to two macaque monkeys and found that, in ECoG recordings over awake macaque areas V1 and V4, the strongest gamma power was induced by images of a colored orange on a gray background. In another study of macaque $\mathrm{V} 1$ responses to natural stimuli, induced gamma power increased with both negative and positive L-M cone contrast (Peter et al., 2020).

In intracortical recordings from macaque V1 under visual stimulation with a peripheral colored disk, Peter et al. (2019) found strongest gamma responses to pink and red stimuli. When colors were chosen from an equiluminance plane in DKL space for a control analysis in one monkey, they still found slightly stronger gamma power for red than for green stimuli, even if red and green stimuli were of the same color contrast. The same asymmetry on the $S-(L+M)$ axis as reported here was found, in the sense that pure blue stimuli induced very weak gamma power. In addition, they explored the effects of prestimulus and background adaptation and found that red induced the strongest gamma power only when presented on a gray background, but not when presented on a black background. Presenting a series of different stimulus and background combinations and summarizing results in a self-defined cone adaptation model, they showed that red and green stimuli induce equal gamma power when the background does not adapt $\mathrm{M}$ - and L- cones, and that, over all stimulus/background combinations, $\mathrm{M}$ cone-activating backgrounds boost L-cone-stimulus-driven gamma oscillations the strongest. This fact suggests that the red effect reported in other studies might be driven by the use of a gray background/inter-trial interval color. 
In recordings from ECoG electrodes implanted over areas V1-V4 in 10 epilepsy patients, Bartoli et al. (2019) found strongest gamma-power responses for full-screen red/orange stimuli following a gray inter-trial interval background. The presented colors were equiluminant and equicontrast in CIELAB, a color coordinate system based on perceptual similarity. In ECoG and intracranial EEG recordings from a second set of 7 epilepsy patients using the same task and stimuli (Li et al., 2021), strongest gammapower responses were found for red/orange and green stimuli. Color gamma responses reported in both studies were of similar strength (expressed in percent change from baseline) as in our MEG study.

Oscillatory neuronal responses to full-screen colored stimuli have also been reported in MEG recordings from 20 participants (Perry et al., 2020). When full-screen red, green, purple, and blue colors were shown after a gray background or in alteration, red stimuli induced the strongest power in several oscillatory bands above $30 \mathrm{~Hz}$.

In total, all previous studies not explicitly controlling for cone contrast found stronger gamma-power responses for red stimuli than for other colors. When cone contrast was controlled for in two macaque V1 studies, either through the use of DKL coordinates or through a self-defined cone adaption model, the difference between red and green stimuli either decreased (Peter et al., 2019; Shirhatti and Ray, 2018) or, when adaptation was also taken into account, disappeared (Peter et al., 2019).

\section{Lower responses for short-wavelength stimuli}

In our data and in a previous report (Peter et al., 2019), induced gamma power was not equal between equicontrast stimuli on the $S-(L+M)$ axis: Blue stimuli showed no or very weak gamma-power increases. Similarly, we found that change-detection performance was worse and ERF amplitudes were smaller. This asymmetry on the $\mathrm{S}-(\mathrm{L}+\mathrm{M})$ axis might be driven by underlying physiology: Compared to $\mathrm{L}-$ and $\mathrm{M}$ cones, S-cones are sparser (de Monasterio et al., 1981) and show slower light responses, higher noise levels, and higher activation thresholds (Baudin et al., 2019; Cole et al., 1993; Lee et al., 2009). Additionally, S-(L+M)-sensitive neurons are sparser than L-M-sensitive ones in the LGN (Derrington et al., 1984). Within V1, neurons sensitive to S-cone inputs reside in different cortical layers and respond later than other color-sensitive neurons (Cottaris and De Valois, 1998). Likely, S-cone-induced signals are comparatively weak within LGN and are amplified and transformed in V1 (De Valois et al., 2000; Xiao, 2013).

The power of V1 gamma oscillations is strongly influenced by the strength of input drive, both in empirical studies (Hadjipapas et al., 2015; Henrie and Shapley, 2005; Jia et al., 2013; Lewis et al., 2021; Roberts et al., 2013) and in computational models (Battaglia and Hansel, 2011; Brunel and Wang, 2003; Jia et al., 2013; Lowet et al., 2015; Mazzoni et al., 2008). Thereby, weak gamma oscillations for stimuli high on the S-(L+M) axis could be explained by the fact that S-cone signals from LGN are carried by a smaller and less strongly driving population of LGN neurons than L-/M-signals. Additionally, late (>85 ms) V1 spiking responses to S-cone input (Cottaris and De Valois, 1998) could explain smaller N70 amplitudes to these stimuli.

Both the source localization and the dependence on input drive (contrast) are features that colorinduced gamma responses share with luminance-contrast induced gamma responses. This points to common principles in the generation of gamma for both chromatic and achromatic stimuli. Yet, the fact that colored disks as compared to gratings induced overall weaker gamma but at the same time more often a second, higher-frequency gamma peak, suggests differences in underlying circuits and/or dynamics. 


\section{A potential source of $\mathbf{V} 1$ gamma selectivity across color hues}

In sum, color-induced gamma responses in area V1 match LGN-to-V1 input strength in three measured aspects: 1 ) along the L-M axis, stimuli driving LGN to an equal degree induce gamma oscillations that do not differ measurably in strength across the population; 2) gamma oscillations are strongly diminished for S-cone-driving stimuli, which are encoded by smaller populations of LGN neurons and which provide comparatively weak excitatory input into $\mathrm{V} 1 ; 3$ ) the amplitude of the early, input-driven N70 component is correlated with later induced gamma power over stimuli. Previous reports of high color specificity of V1 gamma oscillations might therefore be explained by a positive relationship between the strength of feedforward input into V1 and the power of V1 gamma oscillations. 


\section{METHODS}

\section{Participants}

30 participants were recruited from the general public, 18 of them female. They were between 18 and 36 years old (average: 26 years). As participants were recruited via public advertisements, most of them had not participated in vision-science experiments before. They were screened to be free of metal implants, did not take medication during the study period except for contraceptives, had never been diagnosed with any neurological or psychological disorder, had normal or corrected-to-normal vision and did not show red-green color vision deficiencies. The screening criterion for red-green deficiencies was correct answer to all of a nine-plate subset of the 38-plate Ishihara test (Ishihara, 1979). A further five subjects were recruited but excluded due to equipment malfunctions or excessive movements $(>5 \mathrm{~mm})$ during the experiment. The study was approved by the ethics committee of the medical faculty of the Goethe University Frankfurt (Resolution E 36/18).

\section{Paradigm}

Participants were seated in a dark, magnetically shielded, sound-dampened room. Stimuli were shown on a backprojection screen with a distance of $58 \mathrm{~cm}$ to their eyes using a Propixx projector (Resolution: 960*520 px, Refresh rate: $480 \mathrm{~Hz}$ ), controlled with Psychtoolbox-3 (Kleiner et al., 2007). Eye position and pupil size was recorded using an infrared eye tracking system (EyeLink 1000). Before the experiment, participants were trained to minimize saccades and blinks during the baseline and trial periods.

The 540 total trials of the experiment were split into two blocks, with 240 color ( 30 per color) and 30 grating trials shown during each block. Between blocks, participants were given a short break of no longer than five minutes. Within each block, the trial order was chosen randomly for each participant.

A grey background and a dark-grey fixation spot was shown throughout the experiment. Each trial was initiated once the participant fixated the central fixation spot. After a baseline of 1.2 seconds, the stimulus was shown as a central $10 \mathrm{dva}$ diameter disk with broadly anti-aliased, smoothed edges. After a per-trial randomly chosen period of $0.3-2 \mathrm{~s}$ (randomized, Cauchy-distributed with $\mathrm{x}_{0}=1.65 \mathrm{~s}$, FWHM $=0.2 \mathrm{~s}$ ), a to-be-detected local color change (a circular, Gaussian-shaped color step towards the background color, 3.7 dva diameter) was shown on a random position on the stimulus. Participants were instructed to speedily report the left-right position of the target relative to the fixation dot using a button press (left thumb to indicate left, right thumb to indicate right). The relative transparency of the target to the stimulus was QUEST-staircased (Kleiner et al., 2007; Watson and Pelli, 1983) for each stimulus condition and participant to $85 \%$ correct responses. After a button press or after $1 \mathrm{~s}$, the trial was terminated and, if the correct button had been pressed, a smiley was shown for $0.5 \mathrm{~s}$. A random $5 \%$ of trials were target-free catch trials.

\section{Stimuli}

Participants were shown equiluminant colors on an isoluminant background as well as a grating stimulus. To define equiluminance, the projector base-color spectral power distributions were measured using an Ocean Insight FLAME-T spectrometer and used to define per-color cone excitation values using human cone spectral sensitivities for 10 dva diameter stimuli (Stockman and Sharpe, 2000; Stockman et al., 1999). Then, cone excitation values of the neutral gray background and the 
stimuli themselves were used to define per-color coordinates in DKL color space, an opponent modulation space originally developed to describe responses of LGN neurons to color stimuli (Derrington et al., 1984; Westland et al., 2012). $\mathrm{k}_{\mathrm{Lum}}, \mathrm{k}_{\mathrm{L}-\mathrm{M}}$ and $\mathrm{k}_{\mathrm{S}-\mathrm{L}+\mathrm{M}}$ were chosen such that on-axis stimuli with unit pooled cone contrast give unit coordinates in the three DKL axes (Brainard, 1996). The eight color stimuli were chosen from the DKL equiluminance plane to a common, grey background and were chosen to be distributed along an ellipse filling the projector color gamut with equal distances between the color coordinates along the ellipse. For the grating condition, a $1.5 \mathrm{cycle} / \mathrm{dva}$ anti-aliased square wave grating rotated 22.5 deg clockwise from vertical was used.

\section{MEG recording}

Data were recorded using a CTF Systems 275 axial gradiometer MEG system, low-pass filtered $(300 \mathrm{~Hz})$ and digitized $(1200 \mathrm{~Hz})$. Initial head position was set to minimize distance between the occipital pole and posterior MEG gradiometers. Head position was monitored continuously throughout the experiment, and experiment sessions were aborted and excluded from analysis when participants moved their head more than $5 \mathrm{~mm}$ from its initial position. Flexible head fixation using memory foam cushions and medical tape was used throughout the experiment.

\section{Data analysis}

Data were analyzed using custom Matlab and R code and the Fieldtrip toolbox (Oostenveld et al., 2011). Line noise was removed using DFT filters at $50 \mathrm{~Hz}$ and its higher harmonics. The recording was cut into trials from $-1 \mathrm{~s}$ to $1.3 \mathrm{~s}$ relative to stimulus onset. Trials with stimulus changes before $1.3 \mathrm{~s}$ after stimulus onset, trials with missing/early responses, and catch trials were removed. Blink, muscle and SQUID-jump artifacts were detected using a semi-automated artifact detection process. For further analysis, trials were segmented into epochs as detailed below, and analyses were only run for epochs devoid of artifacts. The described trial removal and artifact epoch rejection procedures rejected $19 \%$ of all trials.

\section{Source localization}

Analyses localizing power at the participant-specific gamma peak used dynamic imaging of coherent sources beamforming (Gross et al., 2001). For other analyses, linearly constrained minimum variance (LCMV) beamforming (Van Veen et al., 1997) was used to generate virtual dipole timecourses for all analyzed dipoles. For both beamformers, the covariance matrix was not regularized $(\lambda=0 \%)$ and dipoles were placed at all vertices of both hemispheres of the 32k HCP-MMP1.0 atlas (Glasser et al., 2016a). The atlas was registered to subject-specific T1- and T2-weighted MRI scans (T1: $1 \mathrm{~mm}^{3}$ MPRAGE with TR $=2530 \mathrm{~ms}$, TE $=2.27 \mathrm{~ms} ; \mathrm{T} 2: 1 \mathrm{~mm}^{3} \mathrm{TSE}$ with TR $=1500 \mathrm{~ms}$ and TE $=356 \mathrm{~ms}$, acquired on a 3T Siemens Magnetom Prisma) using Freesurfer (Fischl, 2012) and HCP Workbench (Glasser et al., 2016b). Area-specific analyses (e.g. analyses focusing on area V1) averaged results over all dipoles within the specific area of the atlas. For the calculation of full spectra, to reduce computational demand, a reduced MMP1.0-atlas subsampled to $6.25 \%$ of all 64000 dipole positions was used. Virtual dipole timecourses were computed by multiplying the sensor-level data with the LCMV filters.

\section{Behavioral, ERF and spectral analysis}

Per-stimulus change detection performance was defined as the target-change color contrast the perstimulus staircase had converged to, averaged over the last ten presentations of each stimulus. 
To analyze source-localized ERFs, we low-pass filtered the V1 virtual dipole timecourses using an acausal Gaussian filter kernel $(-6 \mathrm{~dB}$ at $80 \mathrm{~Hz})$, cut them to $-0.2 \mathrm{~s}$ to $0.6 \mathrm{~s}$ relative to stimulus onset and $\mathrm{z}$-scored them relative to the per-trial baseline. Then, we averaged over all V1 dipoles and trials of the same stimulus type within participants. Because the ERF polarity is dependent on dipole orientation and not consistent throughout V1, we flipped (per participant and dipole, yet identical for all stimuli) virtual dipole ERFs that were positive-going during the N70 component ( $55-95 \mathrm{~ms}$ post stimulus onset) before averaging.

For spectral analyses, virtual dipole timecourses were cut into baseline (-1.0 s to 0 s relative to stimulus onset) and stimulus ( $0.3 \mathrm{~s}$ to $1.3 \mathrm{~s}$ after stimulus onset) periods. Then, they were segmented into $50 \%$ overlapping time epochs of $500 \mathrm{~ms}$ (for frequencies $<=20 \mathrm{~Hz}$ ) or $333 \mathrm{~ms}$ length (for frequencies $>20 \mathrm{~Hz}$ ), demeaned and detrended. They were then Hann-tapered (for frequencies $<=20 \mathrm{~Hz}$ ) or Slepian-window multitapered using three tapers to achieve $\pm 3 \mathrm{~Hz}$ smoothing (for frequencies $>2 \mathrm{~Hz}$ ), zero-padded to $1 \mathrm{~s}$ and Fourier transformed. Those Fourier spectra were used to obtain source-level power spectra. Spectra of stimulus-induced power change were computed relative to the per-stimulus averaged baseline spectra and then averaged over the selected dipoles. For time-frequency analyses, the same pipeline with epochs centered on each timepoint of the virtual dipole timecourse (sampling rate $=1200 \mathrm{~Hz}$ ) and zero-padding to $4 \mathrm{~s}$ was run. To define per-trial power change values, we extracted the frequency bin centered on the per-participant, per-stimulus gamma-peak frequency.

To determine per-stimulus, per-participant gamma-peak frequencies, the trial-average spectra of stimulus-induced power change from $15-120 \mathrm{~Hz}$ were fit with three model alternatives: 1) A first-order polynomial. 2) The sum of a first-order polynomial and a Gaussian. 3) The sum of a first-order polynomial and two Gaussians. Spectral bins were weighted according to their scaled power change, and both Gaussians were set to have a minimum amplitude of $5 \%$ and a standard deviation between 1.5-10 Hz. For alternative 2), the single Gaussian was set to have a mean above 21; for alternative 3), one Gaussian was set to have a mean above $21 \mathrm{~Hz}$, and the second Gaussian was set to have a mean above $61 \mathrm{~Hz}$. The model giving the highest adjusted $\mathrm{R}^{2}$ was chosen, and gamma-peak frequencies of the found peaks were extracted. If no gamma-peak frequency was found for a given participantstimulus pair, analyses that required a gamma-peak frequency (DICS beamforming and extracting power at the gamma peak) were computed using the average gamma-peak frequency over stimuli within that participant. These participant-stimulus combinations were excluded from analyses explicitly reporting peak frequency values.

To correlate per-color average ERFs and induced spectra with each other and with behavioral performance, we averaged per-stimulus V1 ERFs and per-stimulus V1 change spectra over trials within each participant. Then, for each timepoint/frequency bin, the per-stimulus value was correlated to the per-stimulus change detection performance. The results were corrected for multiple comparisons over timepoints/frequency bins using $t_{\max }$ multiple-comparison correction (Blair et al., 1994).

\section{Statistical analysis}

Reported tests were two-tailed, alpha was set to $\alpha=0.05$. To calculate evidence towards the null hypothesis for pairwise comparisons, we computed Bayes factors of $\mathrm{H}_{0}$ over $\mathrm{H}_{1}$ using a prior scale of one (Rouder et al., 2009).

To test for significant differences across colors in measures of interest (ERF amplitudes, induced spectral power changes, behavioral performance, reaction times), we averaged these measures per 
bioRxiv preprint doi: https://doi.org/10.1101/2021.11.22.469550; this version posted November 22, 2021. The copyright holder for this preprint (which was not certified by peer review) is the author/funder, who has granted bioRxiv a license to display the preprint in perpetuity. It is made available under aCC-BY 4.0 International license.

stimulus within participants and then performed RMANOVAs across stimuli. The resulting F- and pvalues were Greenhouse-Geisser-corrected where appropriate. Pairwise comparisons were then computed using t-tests, which were Bonferroni-Holm-corrected for multiple comparisons.

\section{Code and data availability}

Custom preprocessing code, per-trial data and code for statistical analyses are available at https://doi.org/10.5281/zenodo.5578940. 


\section{REFERENCES}

Baillet, S. (2017). Magnetoencephalography for brain electrophysiology and imaging. Nat Neurosci 20, 327-339.

Bartoli, E., Bosking, W., Chen, Y., Li, Y., Sheth, S.A., Beauchamp, M.S., Yoshor, D., and Foster, B.L. (2019). Functionally Distinct Gamma Range Activity Revealed by Stimulus Tuning in Human Visual Cortex. Curr Biol 29, 3345-3358 e3347.

Bartoli, E., Bosking, W., and Foster, B.L. (2020). Seeing Visual Gamma Oscillations in a New Light. Trends Cogn Sci 24, 501-503.

Bastos, A.M., Briggs, F., Alitto, H.J., Mangun, G.R., and Usrey, W.M. (2014). Simultaneous recordings from the primary visual cortex and lateral geniculate nucleus reveal rhythmic interactions and a cortical source for gamma-band oscillations. J Neurosci 34, 7639-7644.

Bastos, A.M., Vezoli, J., Bosman, C.A., Schoffelen, J.M., Oostenveld, R., Dowdall, J.R., De Weerd, P., Kennedy, H., and Fries, P. (2015). Visual areas exert feedforward and feedback influences through distinct frequency channels. Neuron 85, 390-401.

Battaglia, D., and Hansel, D. (2011). Synchronous Chaos and Broad Band Gamma Rhythm in a Minimal Multi-Layer Model of Primary Visual Cortex. PLoS Comput Biol 7.

Baudin, J., Angueyra, J.M., Sinha, R., and Rieke, F. (2019). S-cone photoreceptors in the primate retina are functionally distinct from $L$ and $M$ cones. eLife 8, e39166.

Bauer, M., Oostenveld, R., Peeters, M., and Fries, P. (2006). Tactile spatial attention enhances gammaband activity in somatosensory cortex and reduces low-frequency activity in parieto-occipital areas. J Neurosci 26, 490-501.

Benjamini, Y., and Yekutieli, D. (2001). The control of the false discovery rate in multiple testing under dependency. The Annals of Statistics 29, 1165-1188.

Besserve, M., Lowe, S.C., Logothetis, N.K., Schölkopf, B., and Panzeri, S. (2015). Shifts of Gamma Phase across Primary Visual Cortical Sites Reflect Dynamic Stimulus-Modulated Information Transfer. PLoS Biol 13, e1002257.

Blair, R.C., Higgins, J.J., Karniski, W., and Kromrey, J.D. (1994). A Study of Multivariate Permutation Tests Which May Replace Hotelling's T2 Test in Prescribed Circumstances. Multivariate Behavioral Research 29, 141-163.

Bosman, C.A., Schoffelen, J.M., Brunet, N., Oostenveld, R., Bastos, A.M., Womelsdorf, T., Rubehn, B., Stieglitz, T., De Weerd, P., and Fries, P. (2012). Attentional stimulus selection through selective synchronization between monkey visual areas. Neuron $75,875-888$.

Brainard, D.H. (1996). Cone contrast and opponent modulation color space. In Human Color Vision, P.K. Kayser, and R. Boynton, eds. (Washington, DC: Optical Society of America), pp. 563-579.

Brunel, N., and Wang, X.-J. (2003). What Determines the Frequency of Fast Network Oscillations With Irregular Neural Discharges? I. Synaptic Dynamics and Excitation-Inhibition Balance. J Neurophysiol 90, 415-430. 
bioRxiv preprint doi: https://doi.org/10.1101/2021.11.22.469550; this version posted November 22, 2021. The copyright holder for this preprint (which was not certified by peer review) is the author/funder, who has granted bioRxiv a license to display the preprint in perpetuity. It is made available under aCC-BY 4.0 International license.

Brunet, N., Bosman, C.A., Roberts, M., Oostenveld, R., Womelsdorf, T., De Weerd, P., and Fries, P. (2015). Visual cortical gamma-band activity during free viewing of natural images. Cereb Cortex 25, 918-926.

Brunet, N., and Fries, P. (2019). Human visual cortical gamma reflects natural image structure. Neurolmage 200, 635-643.

Brunet, N.M., Bosman, C.A., Vinck, M., Roberts, M., Oostenveld, R., Desimone, R., De Weerd, P., and Fries, P. (2014). Stimulus repetition modulates gamma-band synchronization in primate visual cortex. PNAS 111, 3626-3631.

Butler, R., Bernier, P.-M., Mierzwinski, G.W., Descoteaux, M., Gilbert, G., and Whittingstall, K. (2019). Cortical distance, not cancellation, dominates inter-subject EEG gamma rhythm amplitude. Neurolmage 192, 156-165.

Chatterjee, S., and Callaway, E.M. (2003). Parallel colour-opponent pathways to primary visual cortex. Nature 426, 668-671.

Cole, G.R., Hine, T., and Mcllhagga, W. (1993). Detection mechanisms in L-, M-, and S-cone contrast space. Journal of the Optical Society of America A 10.

Cottaris, N.P., and De Valois, R.L. (1998). Temporal dynamics of chromatic tuning in macaque primary visual cortex. Nature 395, 896-900.

de Monasterio, F.M., Schein, S.J., and McCrane, E.P. (1981). Staining of Blue-Sensitive Cones of the Macaque Retina by a Fluorescent Dye. Science 213, 1278-1281.

De Valois, R.L., Cottaris, N.P., Elfar, S.D., Mahon, L.E., and Wilson, J.A. (2000). Some transformations of color information from lateral geniculate nucleus to striate cortex. Proceedings of the National Academy of Sciences 97, 4997-5002.

Derrington, A.M., Krauskopf, J., and Lennie, P. (1984). Chromatic mechanisms in lateral geniculate nucleus of macaque. J Physiol 357, 241-265.

Felleman, D.J., and Van Essen, D.C. (1991). Distributed hierarchical processing in the primate cerebral cortex. Cereb Cortex 1, 1-47.

Fischl, B. (2012). FreeSurfer. Neurolmage 62, 774-781.

Fries, P. (2015). Rhythms for Cognition: Communication through Coherence. Neuron 88, 220-235.

Fries, P., and Maris, E. (2021). What to do if N is two? arXiv, arXiv:2106.14562 [stat.ME].

Fries, P., Neuenschwander, S., Engel, A.K., Goebel, R., and Singer, W. (2001). Rapid feature selective neuronal synchronization through correlated latency shifting. Nat Neurosci 4, 194-200.

Gieselmann, M.A., and Thiele, A. (2008). Comparison of spatial integration and surround suppression characteristics in spiking activity and the local field potential in macaque V1. Eur J Neurosci 28, 447459.

Glasser, M.F., Coalson, T.S., Robinson, E.C., Hacker, C.D., Harwell, J., Yacoub, E., Ugurbil, K., Andersson, J., Beckmann, C.F., Jenkinson, M., et al. (2016a). A multi-modal parcellation of human cerebral cortex. Nature 536, 171-178. 
bioRxiv preprint doi: https://doi.org/10.1101/2021.11.22.469550; this version posted November 22, 2021. The copyright holder for this preprint (which was not certified by peer review) is the author/funder, who has granted bioRxiv a license to display the preprint in perpetuity. It is made available under aCC-BY 4.0 International license.

Glasser, M.F., Smith, S.M., Marcus, D.S., Andersson, J.L.R., Auerbach, E.J., Behrens, T.E.J., Coalson, T.S., Harms, M.P., Jenkinson, M., Moeller, S., et al. (2016b). The Human Connectome Project's neuroimaging approach. Nat Neurosci 19, 1175-1187.

Gray, C.M., König, P., Engel, A.K., and Singer, W. (1989). Oscillatory responses in cat visual cortex exhibit inter-columnar synchronization which reflects global stimulus properties. Nature 338, 334-337.

Gross, J., Kujala, J., Hamalainen, M., Timmermann, L., Schnitzler, A., and Salmelin, R. (2001). Dynamic imaging of coherent sources: Studying neural interactions in the human brain. PNAS 98, 694-699.

Hadjipapas, A., Lowet, E., Roberts, M.J., Peter, A., and De Weerd, P. (2015). Parametric variation of gamma frequency and power with luminance contrast: A comparative study of human MEG and monkey LFP and spike responses. Neurolmage 112, 327-340.

Henrie, J.A., and Shapley, R. (2005). LFP Power Spectra in V1 Cortex: The Graded Effect of Stimulus Contrast. J Neurophysiol 94, 479-490.

Hermes, D., Petridou, N., Kay, K.N., and Winawer, J. (2019). An image-computable model for the stimulus selectivity of gamma oscillations. eLife 8 , e47035.

Hoogenboom, N., Schoffelen, J.M., Oostenveld, R., Parkes, L.M., and Fries, P. (2006). Localizing human visual gamma-band activity in frequency, time and space. Neurolmage $29,764-773$.

Ishihara, S. (1979). Tests for colour-blindness, 38 plates edition (Tokyo: Kanehara Shuppan Co, Ltd.).

Jia, X., Smith, M.A., and Kohn, A. (2011). Stimulus Selectivity and Spatial Coherence of Gamma Components of the Local Field Potential. J Neurosci 31, 9390-9403.

Jia, X., Xing, D., and Kohn, A. (2013). No consistent relationship between gamma power and peak frequency in macaque primary visual cortex. J Neurosci 33, 17-25.

Kleiner, M., Brainard, D., and Pelli, D. (2007). What's new in Psychtoolbox-3? Perception 36 (ECVP Abstract Supplement), 14.

Kreiter, A.K., and Singer, W. (1992). Oscillatory Neuronal Responses in the Visual Cortex of the Awake Macaque Monkey. Eur J Neurosci 4, 369-375.

Lee, R.J., Mollon, J.D., Zaidi, Q., and Smithson, H.E. (2009). Latency characteristics of the shortwavelength-sensitive cones and their associated pathways. J Vis 9, 5-5.

Lewis, C.M., Ni, J., Wunderle, T., Jendritza, P., Lazar, A., Diester, I., and Fries, P. (2021). Cortical gammaband resonance preferentially transmits coherent input. Cell Reports 35.

Li, Y., Bosking, W., Beauchamp, M.S., Sheth, S.A., Yoshor, D., Bartoli, E., and Foster, B.L. (2021). Orientation and color tuning of the human visual gamma rhythm. bioRxiv.

Lowet, E., Roberts, M., Hadjipapas, A., Peter, A., van der Eerden, J., and De Weerd, P. (2015). Inputdependent frequency modulation of cortical gamma oscillations shapes spatial synchronization and enables phase coding. PLoS Comput Biol 11, e1004072.

Lowet, E., Roberts, M.J., Peter, A., Gips, B., and De Weerd, P. (2017). A quantitative theory of gamma synchronization in macaque $\mathrm{V} 1$. elife 6. 
bioRxiv preprint doi: https://doi.org/10.1101/2021.11.22.469550; this version posted November 22, 2021. The copyright holder for this preprint (which was not certified by peer review) is the author/funder, who has granted bioRxiv a license to display the preprint in perpetuity. It is made available under aCC-BY 4.0 International license.

Magazzini, L., and Singh, K.D. (2018). Spatial attention modulates visual gamma oscillations across the human ventral stream. Neurolmage 166, 219-229.

Mazzoni, A., Panzeri, S., Logothetis, N.K., and Brunel, N. (2008). Encoding of Naturalistic Stimuli by Local Field Potential Spectra in Networks of Excitatory and Inhibitory Neurons. PLoS Comput Biol 4.

Michalareas, G., Vezoli, J., van Pelt, S., Schoffelen, J.M., Kennedy, H., and Fries, P. (2016). Alpha-Beta and Gamma Rhythms Subserve Feedback and Feedforward Influences among Human Visual Cortical Areas. Neuron 89, 384-397.

Murakami, S., and Okada, Y. (2006). Contributions of principal neocortical neurons to magnetoencephalography and electroencephalography signals. J Physiol 575, 925-936.

Nasiotis, K., Clavagnier, S., Baillet, S., and Pack, C.C. (2017). High-resolution retinotopic maps estimated with magnetoencephalography. Neurolmage 145, 107-117.

Neitz, J., and Neitz, M. (2011). The genetics of normal and defective color vision. Vision Res 51, 633651.

Oostenveld, R., Fries, P., Maris, E., and Schoffelen, J.M. (2011). FieldTrip: Open source software for advanced analysis of MEG, EEG, and invasive electrophysiological data. Comput Intell Neurosci 2011, 156869.

Perry, G., Taylor, N.W., Bothwell, P.C.H., Milbourn, C.C., Powell, G., and Singh, K.D. (2020). The gamma response to colour hue in humans: Evidence from MEG. PLoS One 15.

Peter, A., Stauch, B.J., Shapcott, K., Kouroupaki, K., Schmiedt, J.T., Klein, L., Klon-Lipok, J., Dowdall, J.R., Schölvinck, M.L., Vinck, M., et al. (2020). Stimulus-specific plasticity of macaque V1 spike rates and gamma. bioRxiv.

Peter, A., Uran, C., Klon-Lipok, J., Roese, R., van Stijn, S., Barnes, W., Dowdall, J.R., Singer, W., Fries, P., and Vinck, M. (2019). Surface color and predictability determine contextual modulation of V1 firing and gamma oscillations. eLife 8 , e42101.

Roberts, M.J., Lowet, E., Brunet, N.M., Ter Wal, M., Tiesinga, P., Fries, P., and De Weerd, P. (2013). Robust gamma coherence between macaque V1 and V2 by dynamic frequency matching. Neuron 78 , 523-536.

Rols, G., Tallon-Baudry, C., Girard, P., Bertrand, O., and Bullier, J. (2001). Cortical mapping of gamma oscillations in areas V1 and V4 of the macaque monkey. Vis Neurosci 18, 527-540.

Rouder, J.N., Speckman, P.L., Sun, D., Morey, R.D., and Iverson, G. (2009). Bayesian t tests for accepting and rejecting the null hypothesis. Psychonomic Bulletin \& Review 16, 225-237.

Shapley, R. (2019). Physiology of Color Vision in Primates. In Oxford Research Encyclopedia of Neuroscience.

Sherman, S.M. (2005). Thalamic relays and cortical functioning. In Cortical Function: a View from the Thalamus, pp. 107-126.

Shirhatti, V., and Ray, S. (2018). Long-wavelength (reddish) hues induce unusually large gamma oscillations in the primate primary visual cortex. Proc Natl Acad Sci U S A 115, 4489-4494. 
Singer, W. (2018). Neuronal oscillations: unavoidable and useful? European Journal of Neuroscience 48, 2389-2398.

Stauch, B.J., Peter, A., Schuler, H., and Fries, P. (2021). Stimulus-specific plasticity in human visual gamma-band activity and functional connectivity. eLife 10, e68240.

Stockman, A., and Sharpe, L.T. (2000). The spectral sensitivities of the middle- and long-wavelengthsensitive cones derived from measurements in observers of known genotype. Vision Res 40, 17111737.

Stockman, A., Sharpe, L.T., and Fach, C. (1999). The spectral sensitivity of the human short-wavelength sensitive cones derived from thresholds and color matches. Vision Res 39, 2901-2927.

Uran, C., Peter, A., Lazar, A., Barnes, W., Klon-Lipok, J., Shapcott, K.A., Roese, R., Fries, P., Singer, W., and Vinck, M. (2020). Predictability in natural images determines V1 firing rates and synchronization: A deep neural network approach. bioRxiv.

van Kerkoerle, T., Self, M.W., Dagnino, B., Gariel-Mathis, M.A., Poort, J., van der Togt, C., and Roelfsema, P.R. (2014). Alpha and gamma oscillations characterize feedback and feedforward processing in monkey visual cortex. Proc Natl Acad Sci U S A.

van Pelt, S., Boomsma, D.I., and Fries, P. (2012). Magnetoencephalography in twins reveals a strong genetic determination of the peak frequency of visually induced gamma-band synchronization. J Neurosci 32, 3388-3392.

van Pelt, S., Shumskaya, E., and Fries, P. (2018). Cortical volume and sex influence visual gamma. Neurolmage 178, 702-712.

Van Veen, B.D., Van Drongelen, W., Yuchtman, M., and Suzuki, A. (1997). Localization of brain electrical activity via linearly constrained minimum variance spatial filtering. IEEE Trans Biomed Eng 44, 867-880.

Vinck, M., and Bosman, C.A. (2016). More Gamma More Predictions: Gamma-Synchronization as a Key Mechanism for Efficient Integration of Classical Receptive Field Inputs with Surround Predictions. Front Syst Neurosci 10, 35.

Watson, A.B., and Pelli, D.G. (1983). Quest: A Bayesian adaptive psychometric method. Perception \& Psychophysics 33, 113-120.

Westland, S., Ripamonti, C., and Cheung, V. (2012). Computational colour science using MATLAB, 2nd edn (Hoboken, NJ: Wiley).

Xiao, Y. (2013). Processing of the S-cone signals in the early visual cortex of primates. Vis Neurosci 31, 189-195. 


\section{ACKNOWLEDGMENTS}

We thank Karl R. Gegenfurtner, Jarrod R. Dowdall, Cem Uran, and Joscha T. Schmiedt for insightful discussions about color spaces. We also thank the ESI workshop and IT teams as well as the BIC MEG and MRI infrastructure groups for their support.

\section{AUTHOR CONTRIBUTIONS}

Conceptualization: B.J.S., A.P. and P.F.; Formal analysis: B.J.S. and Z.N.; Funding acquisition: P.F.; Investigation: B.J.S. and I.E.; Software: B.J.S. and Z.N.; Supervision: P.F.; Visualization: B.J.S.; Writing original draft: B.J.S.; Writing - review \& editing: B.J.S., A.P. and P.F.

\section{DECLARATION OF INTERESTS}

P.F. has a patent on thin-film electrodes (US20170181707A1) and is beneficiary of a respective license contract on thin-film electrodes with Blackrock Microsystems LLC (Salt Lake City, UT). P.F. is member of the Scientific Technical Advisory Board of CorTec GmbH (Freiburg, Germany), and managing director of Brain Science GmbH (Frankfurt am Main, Germany). The authors declare no further competing interests. 


\section{Figure titles and legends}

Figure 1. Experiment design: (A) Temporal structure of one trial. After fixation onset, a grey baseline was shown for $1.2 \mathrm{~s}$, followed by $0.3-2 \mathrm{~s}$ of stimulation with a stimulus disk. Then, a change spot appeared at a random position on the disk (indicated here with an arrow, not visible in the actual experiment), which the participants needed to report. Upon correct report, a smiley was shown. (B) Human cone sensitivity spectra (Stockman and Sharpe, 2000) plotted on an estimate of perceptual wavelength color. (C) DKL coordinates for all eight stimulus colors shown in this study, relative to the background. Letters indicate stimulus labels used in Results. Note that color hues of these color disks, when displayed on a non-calibrated monitor or printout, will not fully correspond to the hues shown in the study. (D) To compare the strength of induced gamma responses between color and grating stimuli, some trials showed a grating instead of a color stimulus.

Figure 2. Event-related field responses: (A) Grating-evoked ERF, averaged over V1 dipoles and participants. Error region shows $95 \% \mathrm{Cl}$ over participants. (B) Color-evoked ERFs, averaged over V1 dipoles and participants. Black bar indicates significant differences across colors, $t_{\max }$-corrected for multiple comparisons. Line colors reflect stimulus colors, but have been darkened for readability. (C) ERF dipole moment 70-90 ms post-stimulus onset (relative to baseline variability), averaged over V1 dipoles and participants. Error bars represent $95 \% \mathrm{Cls}$ over participants, bar orientation represents stimulus orientation in DKL space. In grey, the average grating-induced ERF dipole moment is shown for comparison. (D) Average relative contrast steps towards the background color needed to achieve 85\% target detection accuracy for all stimuli. Error bars represent $95 \%$ Cls over participants, bar orientation represents stimulus orientation in DKL space. For C-D, the length of the scale bar corresponds to the distance from the edge of the hexagon to the outer ring.

Figure 3. Gamma-band responses: (A) Stimulus-induced power changes over baseline for one example participant that showed strong gamma-band responses (averaged over V1 dipoles). Top: Gratinginduced power changes. Error region shows $95 \% \mathrm{Cl}$ over trials. Bottom: Color-induced power changes. (B) Same as A, but averaged over participants. Error region shows $95 \% \mathrm{Cl}$ over participants. (C) Same as $B$, but peak-aligned before averaging. For $A-C$, line colors reflect stimulus colors, but have been darkened for readability. (D) Average stimulus-induced power change in V1 as a function of time and frequency. Top: For grating stimuli. Bottom: For green stimuli. (E) Average stimulus-induced gamma power change for all stimuli. Error bars represent $95 \% \mathrm{Cls}$ over participants, bar orientation represents stimulus orientation in DKL space. In grey, the average grating-induced gamma power strength is shown for comparison. The length of the scale bar corresponds to the distance from the edge of the hexagon to the outer ring. (F) Average stimulus-induced gamma-power change (individual gamma peak $\pm 10 \mathrm{~Hz}$ ), source-projected to all cortical dipoles. Values are significance-masked using false discovery rate control (Benjamini and Yekutieli, 2001), black-to-white shading indicates areas V1, V2, V3, V3A, and V4. (G) Gamma peak frequencies of the first and second gamma peak for all participantcolor combinations in which a first and a second gamma peak was found. Dot color corresponds to stimulus color. Dotted line indicates the expected frequency relationship, if $1^{\text {st }}$ and $2^{\text {nd }}$ peak frequencies were harmonics of each other.

Figure 4. Correlations between ERF, induced power spectra and performance: (A) Correlation, across colors, between V1 ERF dipole moment (averaged over trials and dipoles) and 85\% accuracy threshold. The correlation was first calculated per participant and then averaged over participants. Error-bands represent $95 \%$ Cls over participants. In grey, the average ERF dipole moment timecourse over color 
stimuli is shown for comparison. Horizontal blue bar represents significant correlation values (multiple comparison-corrected using $t_{\max }$ correction). (B) Same as A, but for the stimulus-induced V1 power change spectra instead of the ERFs. (C) Correlation, across colors, between V1 ERF dipole moment and V1 stimulus-induced power change, as a function of ERF time and spectral frequencies. The correlation was first calculated per participant and then averaged over participants. Significant correlation values (multiple comparison-corrected using $\mathrm{t}_{\max }$ correction) are indicated by green dots.

Figure 1-figure supplement 1. Stimulus color coordinates: DKL- (relative to grey background) and CIELAB-coordinates (relative to standard illuminant D65) of the stimuli and the background shown in this study.

Figure 2-figure supplement 1. Reaction time results: Average reaction times for all stimuli. Error bars represent $95 \% \mathrm{Cls}$ over participants, bar orientation represents stimulus orientation in DKL space. The length of the scale bar corresponds to the distance from the edge of the hexagon to the outer ring.

Figure 3-figure supplement 1. Spectral measures: (A) Average induced gamma-peak frequency of the lower gamma peak for all stimuli. Bar orientation represents stimulus orientation in DKL space. In grey, the same is shown for the grating stimulus. (B) Same as A, but for the upper gamma peak frequency. (C) Same as A, but for stimulus-induced power at the upper gamma peak frequency. In all panels, error bars represent $95 \% \mathrm{Cls}$ over participants and the length of the scale bar corresponds to the distance from the edge of the hexagon to the outer ring.

Figure 3-figure supplement 2. Individual participant spectra: Stimulus-induced power changes over baseline (averaged over V1 dipoles) for all 30 participants and the eight presented colors. 


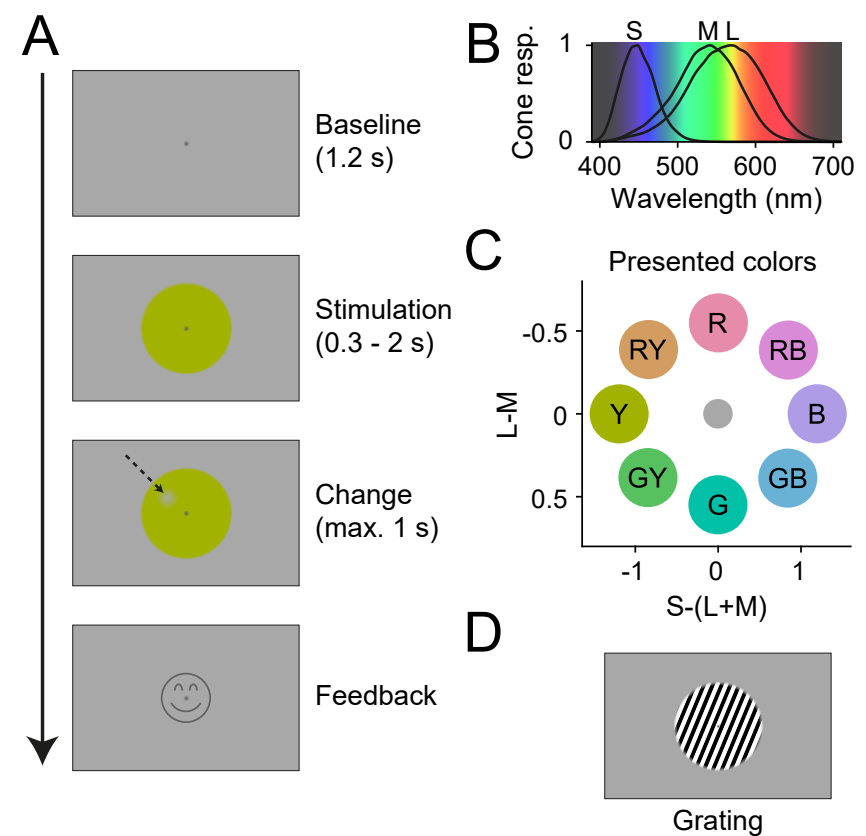

Figure 1. Experiment design: (A) Temporal structure of one trial. After fixation onset, a grey baseline was shown for $1.2 \mathrm{~s}$, followed by $0.32 \mathrm{~s}$ of stimulation with a stimulus disk. Then, a change spot appeared at a random position on the disk (indicated here with an arrow, not visible in the actual experiment), which the participants needed to report. Upon correct report, a smiley was shown. (B) Human cone sensitivity spectra (Stockman and Sharpe, 2000) plotted on an estimate of perceptual wavelength color. (C) DKL coordinates for all eight stimulus colors shown in this study, relative to the background. Letters indicate stimulus labels used in Results. Note that color hues of these color disks, when displayed on a non-calibrated monitor or printout, will not fully correspond to the hues shown in the study. (D) To compare the strength of induced gamma responses between color and grating stimuli, some trials showed a grating instead of a color stimulus.

\begin{tabular}{ccccccc}
\hline Color label & $L+M$ & $L-M$ & $S-(L+M)$ & $L^{*}$ & $a^{*}$ & $b^{*}$ \\
\hline Background & 0.000 & 0.000 & 0.000 & 69.470 & 0.000 & 0.000 \\
Red & -0.008 & 0.549 & 0.003 & 68.548 & 38.335 & -1.035 \\
Red-Blue & -0.001 & 0.385 & 0.848 & 68.574 & 41.473 & -26.561 \\
Blue & -0.002 & 0.000 & 1.195 & 69.062 & 21.859 & -34.510 \\
Green-Blue & -0.002 & -0.388 & 0.840 & 69.776 & -13.640 & -24.862 \\
Green & 0.008 & -0.549 & -0.003 & 70.372 & -46.237 & 1.006 \\
Green-Yellow & 0.001 & -0.385 & -0.848 & 70.347 & -51.207 & 40.356 \\
Yellow & 0.002 & 0.000 & -1.195 & 69.873 & -24.315 & 71.221 \\
Red-Yellow & 0.002 & 0.388 & -0.840 & 69.161 & 12.877 & 39.263 \\
\hline
\end{tabular}

Figure 1-figure supplement 1. Stimulus color coordinates: DKL- (relative to grey background) and CIELAB-coordinates (relative to standard illuminant D65) of the stimuli and the background shown in this study. 
A

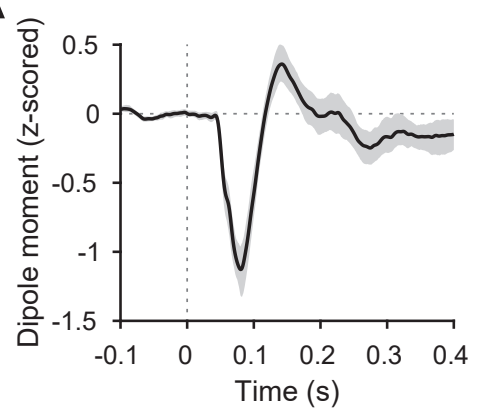

C

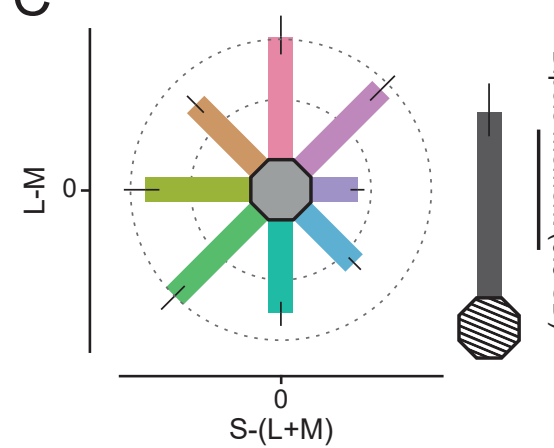

$\mathrm{B}$

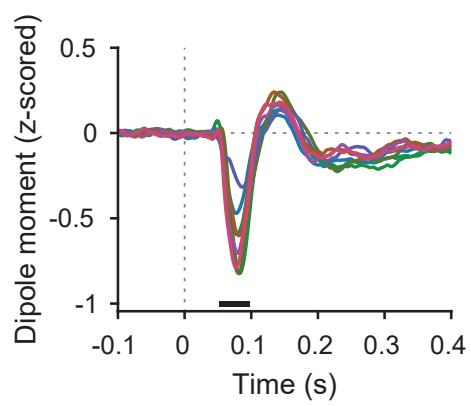

$\mathrm{D}$

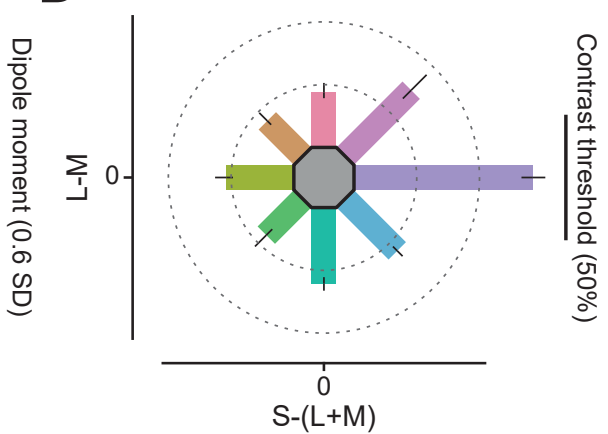

Figure 2. Event-related field responses: (A) Grating-evoked ERF, averaged over V1 dipoles and participants. Error region shows $95 \% \mathrm{Cl}$ over participants. (B) Color-evoked ERFs, averaged over V1 dipoles and participants. Black bar indicates significant differences across colors, $t_{\max }{ }^{-}$corrected for multiple comparisons. Line colors reflect stimulus colors, but have been darkened for readability. (C) ERF dipole moment 70-90 ms post-stimulus onset (relative to baseline variability), averaged over V1 dipoles and participants. Error bars represent $95 \% \mathrm{Cls}$ over participants, bar orientation represents stimulus orientation in DKL space. In grey, the average grating-induced ERF dipole moment is shown for comparison. (D) Average relative contrast steps towards the background color needed to achieve $85 \%$ target detection accuracy for all stimuli. Error bars represent $95 \%$ Cls over participants, bar orientation represents stimulus orientation in DKL space. For C-D, the length of the scale bar corresponds to the distance from the edge of the hexagon to the outer ring. 
bioRxiv preprint doi: https://doi.org/10.1101/2021.11.22.469550; this version posted November 22, 2021. The copyright holder for this

preprint (which was not certified by peer review) is the author/funder, who has granted bioRxiv a license to display the preprint in perpetuity. It is made available under aCC-BY 4.0 International license.

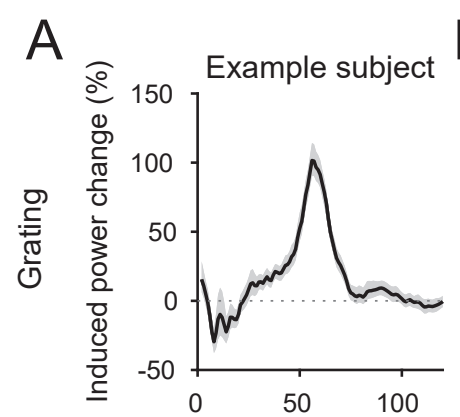

B

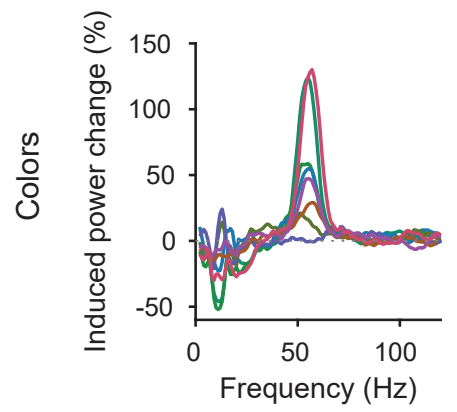

E

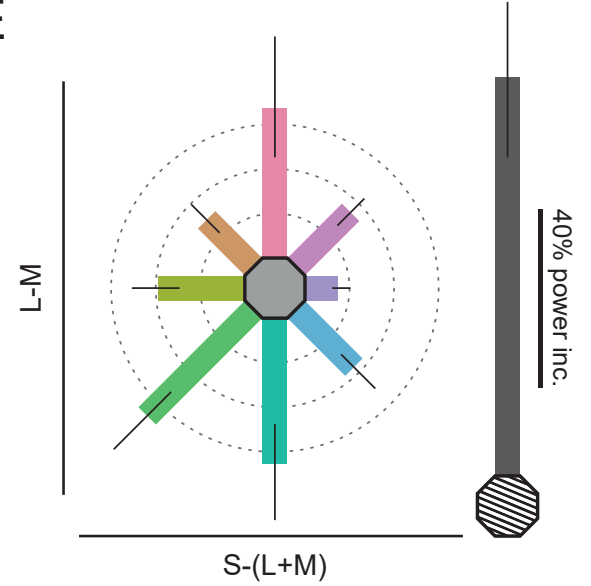

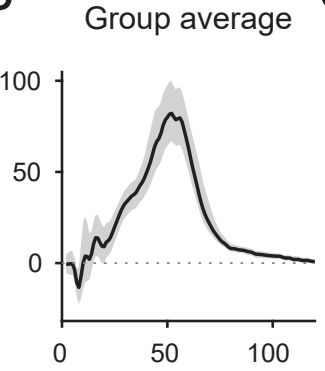

C
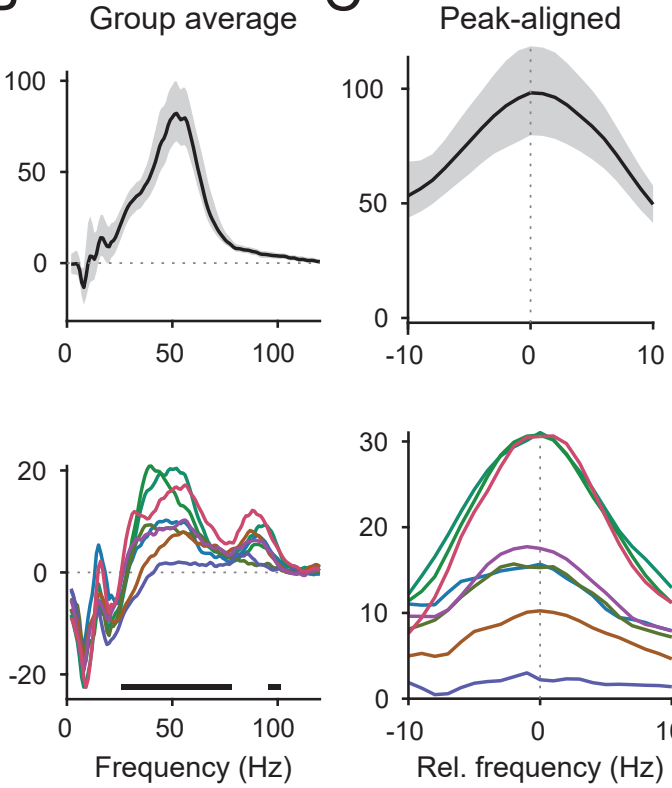

$\mathrm{F}$

Rel. frequency $(\mathrm{Hz})$
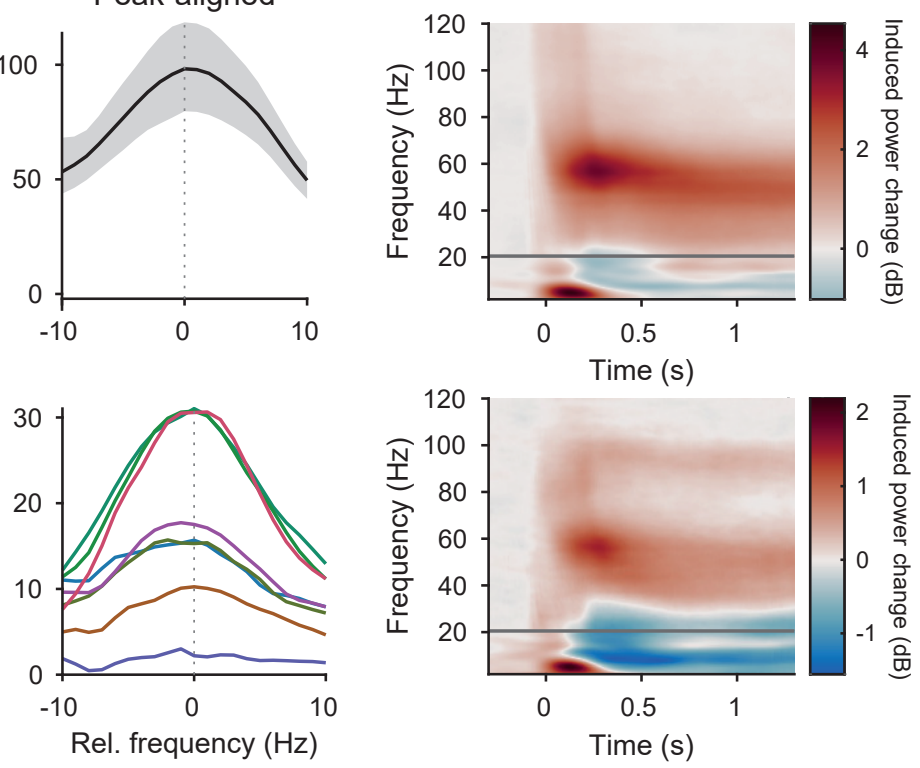

G

Grating
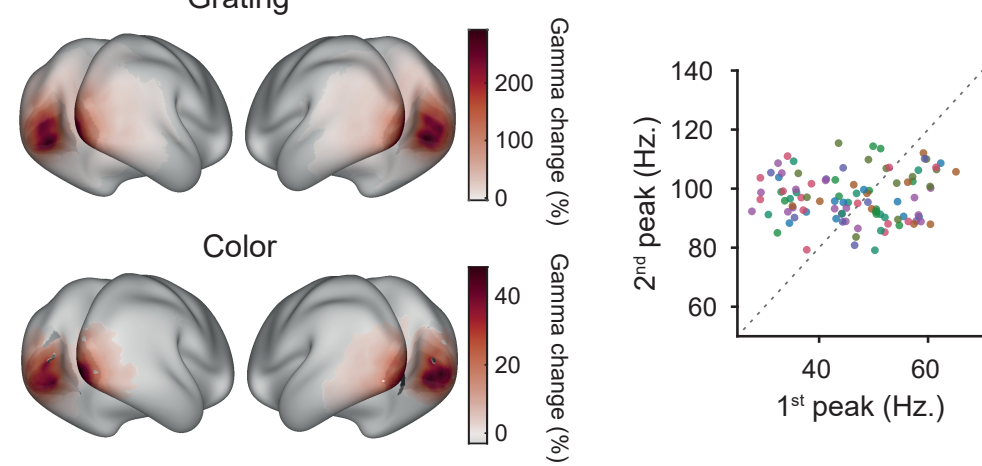

Figure 3. Gamma-band responses: (A) Stimulus-induced power changes over baseline for one example participant that showed strong gamma-band responses (averaged over V1 dipoles). Top: Grating-induced power changes. Error region shows $95 \% \mathrm{Cl}$ over trials. Bottom: Color-induced power changes. (B) Same as A, but averaged over participants. Error region shows $95 \% \mathrm{Cl}$ over participants. (C) Same as B, but peak-aligned before averaging. For $A-C$, line colors reflect stimulus colors, but have been darkened for readability. (D) Average stimulus-induced power change in V1 as a function of time and frequency. Top: For grating stimuli. Bottom: For green stimuli. (E) Average stimulus-induced gamma power change for all stimuli. Error bars represent $95 \% \mathrm{Cls}$ over participants, bar orientation represents stimulus orientation in DKL space. In grey, the average grating-induced gamma power strength is shown for comparison. The length of the scale bar corresponds to the distance from the edge of the hexagon to the outer ring. $(F)$ Average stimulus-induced gamma-power change (individual gamma peak $\pm 10 \mathrm{~Hz}$ ), source projected to all cortical dipoles. Values are significance-masked using false discovery rate control (Benjamini and Yekutieli, 2001), black-to-white shading indicates areas V1, V2, V3, V3A, and V4. (G) Gamma peak frequencies of the first and second gamma peak for all participant-color combinations in which a first and a second gamma peak was found. Dot color corresponds to stimulus color. Dotted line indicates the expected frequency relationship, if 1 st and 2 nd peak frequencies were harmonics of each other. 
A

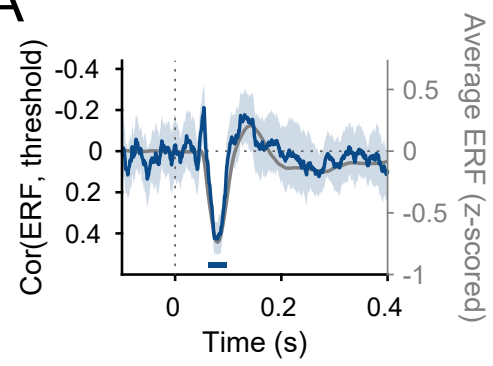

B
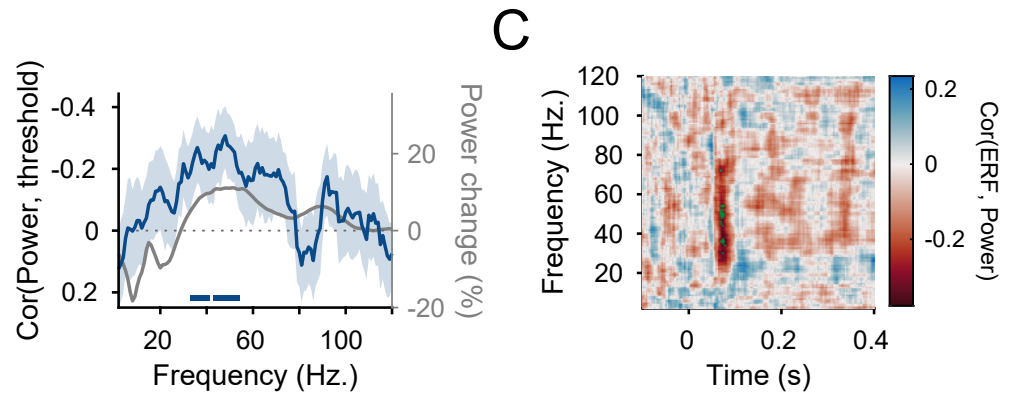

Figure 4. Correlations between ERF, induced power spectra and performance: (A) Correlation, across colors, between V1 ERF dipole moment (averaged over trials and dipoles) and $85 \%$ accuracy threshold. The correlation was first calculated per participant and then averaged over participants. Error-bands represent $95 \% \mathrm{Cls}$ over participants. In grey, the average ERF dipole moment timecourse over color stimuli is shown for comparison. Horizontal blue bar represents significant correlation values (multiple comparison corrected using tmax correction). (B) Same as A, but for the stimulus-induced V1 power change spectra instead of the ERFs. (C) Correlation, across colors, between V1 ERF dipole moment and V1 stimulus-induced power change, as a function of ERF time and spectral frequencies. The correlation was first calculated per participant and then averaged over participants. Significant correlation values (multiple comparison-corrected using tmax correction) are indicated by green dots. 